\title{
Longitudinal Patterns in Survival, Comorbidity, Healthcare Utilization and Quality of Care among Older Women Following Breast Cancer Diagnosis
}

\author{
Amresh D. Hanchate, $P h D^{1,2}$, Kerri M. Clough-Gorr, DSc, MPH ${ }^{3,4,5}$, Arlene S. Ash, PhD 2,6 , \\ Soe Soe Thwin, $P h D^{3,7}$, and Rebecca A. Silliman, $M D, P h D^{3,4}$
}

${ }^{1}$ Center for Organization, Leadership and Management Research (COLMR), VA Boston Healthcare System, Boston, MA, USA; ${ }^{2}$ Health Care Research Unit, Section of General Internal Medicine, Boston University School of Medicine, Boston, MA, USA; ${ }^{3}$ Section of Geriatrics, Boston University School of Medicine, Boston, MA, USA; ${ }^{4}$ Department of Epidemiology, Boston University School of Public Health, Boston, MA, USA; ${ }^{5}$ National Institute for Cancer Epidemiology and Registration (NICER) Institute of Social and Preventative Medicine (ISPM), University of Zurich, Zurich, Switzerland; 'Department of Quantitative Health Sciences, University of Massachusetts Medical School, Worcester, MA, USA; ${ }^{7}$ Massachusetts Veterans Epidemiology Research and Information Center (MAVERIC), VA Healthcare System, Boston, MA, USA.

\begin{abstract}
OBJECTIVES: To compare longitudinal patterns of health care utilization and quality of care for other health conditions between breast cancer-surviving older women and a matched cohort without breast cancer.
\end{abstract}

DESIGN: Prospective five-year longitudinal comparison of cases and matched controls.

SUBJECTS: Newly identified breast cancer patients recruited during 1997-1999 from four geographic regions (Los Angeles, CA; Minnesota; North Carolina; and Rhode Island; $N=422$ ) were matched by age, race, baseline comorbidity and zip code location with up to four non-breast-cancer controls $(N=1,656)$.

OUTCOMES: Survival; numbers of hospitalized days and physician visits; total inpatient and outpatient Medicare payments; guideline monitoring for patients with cardiovascular disease and diabetes, and bone density testing and colorectal cancer screening.

RESULTS: Five-year survival was similar for cases and controls $(80 \%$ and $82 \%$, respectively; $p=0.18)$. In the first follow-up year, comorbidity burden and health care utilization were higher for cases $(p<0.01)$, with most differences diminishing over time. However, the number of physician visits was higher for cases $(p<0.01)$ in every year, driven partly by more cancer and surgical specialist visits. Cases and controls adhered similarly to recommended bone density testing, and monitoring of cardiovascular disease and diabetes; adherence to recommended colorectal cancer screening was better among cases.

CONCLUSION: Breast cancer survivors' health care utilization and disease burden return to pre-diagnosis levels after one year, yet their greater use of outpatient care persists at least five years. Quality of care

Received September 30, 2009

Revised March 31, 2010

Accepted May 12, 2010

Published online June 8, 2010 for other chronic health problems is similar for cases and controls.

KEY WORDS: survival; case-control; inpatient care; outpatient care; costs; preventive care.

J Gen Intern Med 25(10):1045-50

DOI: $10.1007 / \mathrm{s} 11606-010-1407-9$

(c) Society of General Internal Medicine 2010

$\mathrm{D}$ emographics and therapeutic progress in the United States are increasing the number of older cancer survivors. About 182,500 women were diagnosed with breast cancer in 2008, almost half occurring in women aged 65 or older. At five years, $89 \%$ remain alive; ${ }^{1}$ of over 2.6 million breast cancer survivors in the United States, more than half are $\geq 65$ years old. ${ }^{2}$ Both the prevalence and absolute numbers of breast cancer survivors will grow, because aging is the most important risk factor for breast cancer; ${ }^{3}$ gains in life expectancy and advances in detection and treatment will place more women at risk for breast cancer, and breast cancer survivorship.

Comorbidities also increase with age, for those with cancer and others. ${ }^{4-7}$ Across most cancer types, older cancer patients report significantly more comorbidity and poorer physical health than non-cancer patients of the same age. ${ }^{6-8}$ Since hypertension, heart conditions, arthritis, and diabetes are common in older breast cancer survivors, ${ }^{7}$ the Institute of Medicine has emphasized quality follow-up care for this cohort..$^{9,10}$

Although follow-up care is essential in cancer survivorship, ${ }^{11-15}$ studies comparing the quality of care for cancer survivors to those without cancer are conflicting. A longitudinal study of patients with diabetes showed similar quality of diabetes care for patients with and without cancer. ${ }^{16}$ Other studies have found that older cancer survivors receive similar, or less follow-up care than controls, or that the type of primary and cancer-related care varies by provider type. ${ }^{9,11-14}$ Finally, 
a SEER-Medicare study of older breast cancer survivors found them receiving more preventative services than non-cancer patients. ${ }^{17}$ The inconsistent results may be due to different study populations (e.g. source, age, and type of cancer) and/or the kinds of follow-up care examined.

To answer whether overall healthcare utilization and quality of follow-up care for other conditions differs for older women with and without breast cancer, we conducted a multi-site study of women $\geq 65$-years of age with breast cancer and age-, morbidity-, and geography-matched controls. We compared differences in survival and quality of follow-up care over five years.

\section{METHODS}

\section{Study Design}

In this prospective, longitudinal study, we compared survival, comorbidity, healthcare utilization, and quality of care between breast cancer patients and a matched control cohort using Medicare data from the Centers for Medicare and Medicaid Services (CMS). We examined annual comorbidity and healthcare utilization for five years following the date of breast cancer diagnosis. Quality of care was defined as guideline-consistent colorectal cancer screening, bone density testing, and monitoring of cardiovascular disease and diabetes.

\section{Study Participants}

Recruitment procedures for the breast cancer cohort are detailed elsewhere. ${ }^{18}$ Briefly, we identified newly diagnosed breast cancer patients by reviewing pathology reports at hospitals or tumor registries in four geographic regions (Los Angeles, California; Minnesota; North Carolina; and Rhode Island). Each setting received Institutional Review Board (IRB) approval. Eligibility required: stage II-IIIA disease or stage I disease with a tumor diameter $\geq 1 \mathrm{~cm}$; age at diagnosis $\geq 65$ years; no prior history of primary breast cancer; no simultaneously diagnosed or treated second primary tumor at another site; English speaking; and, competent for interview.

Eligible participants were mailed an enrollment package and called by a research staff member. Between 1997 and 1999, 921 women agreed to participate by returning an IRBapproved signed consent form, and were enrolled. Tumor information was collected by medical record review, with date of definitive biopsy treated as the enrollment date for each case. Based on additional information, 56 respondents did not meet inclusion criteria, leaving 865 eligible cases.

Because fee-for-service CMS records were the only source of utilization information for both cases and controls, some otherwise eligible cases could not be included in this comparison study. Such records were only available for Medicare enrollees with both inpatient (Part A) and outpatient (Part B) entitlement and fee-for-service (FFS) coverage. We excluded 301 cases without such coverage for the entire period beginning one year before study enrollment and for five years following. Additional case exclusions were due to problematic Medicare identifiers ( $n=136)$; no matched controls $(n=5)$; and no confirmation of breast cancer diagnosis in the administrative data $(n=1)$.
For the remaining 422 cases, we selected controls in two stages. First, using the full (100\%) Medicare data, we identified all potential controls $(n=21,241)$ that exactly matched at least one case by age, race (White, Black or Other) and 5-digit zip code location. For 5.8\% of cases we did not find exact matches and therefore relaxed the matching criteria for age ( $\pm /-2$-years) and zip code (within 20 -mile radius). We excluded women with a history of breast cancer.

From among the 21,241 potential controls for the 422 cases, we sought four controls for each case-specifically the four non-cases who best matched the comorbidity burden of the case in the year prior to her cancer diagnosis. Selected controls were assigned the same "enrollment date" as its matched case.

"Comorbidity burden" was measured by a prospective diagnostic cost group (DCG) score, calculated from age, sex and all ICD-9-CM diagnosis codes recorded-excluding breast cancer diagnosis codes-for inpatient and outpatient encounters during the year preceding "enrollment." 19 The diagnostic cost group (DCG) risk adjustment system is a validated risk adjustment system. ${ }^{20,21}$ Originally developed for setting prospective Medicare payments, DCG models are now used to risk adjust various outcomes in a range of populations. The DCG score used here indicates the expected future cost of utilization, normalized to average 1.0 in the Medicare over-age-65 population. All but 13 of the 422 cases had at least five potential controls, from which the four potential controls with comorbidity burden (DCG score) nearest to that of the matched case were selected. Of the rest, one had four potential controls; two had three; and ten had one. These were all included in the final study population of 422 cases and 1,656 controls.

\section{Data Sources and Variables}

For the study population, we obtained Medicare eligibility files for 1996 to 2004 and Medicare claims files (MEDPAR, carrier and outpatient) for 1996 to 2003.

We defined outcome measures using the day after the study enrollment date (which differs by case), as the beginning of followup. We identified dates of death through 12/31/2004 from CMS denominator files. We measured comorbidity burden using the DCG score, as above, for each of five 365-day periods subsequent to the study enrollment date. To measure total non-breast cancer illness burden, we dropped breast cancer codes (ICD-9-CM 174.0-174.9, 198.81) before calculating the score. In each year, utilization was measured as follows: overall utilization as total Medicare payments; inpatient, as both 1) number of hospital days and 2) Medicare payments charged for inpatient care: outpatient, as payments for outpatient care (including physician visits, imaging, laboratory tests and procedures). We also examined payments by type of care, ${ }^{22}$ and a narrow outpatient measure: number of physician visits. Using provider specialty codes we categorized visits by specialty - cancer, cardiopulmonary, mental health, surgery, generalist and other. Since outliers among individual expenditures would unduly influence overall statistics, annual inpatient payment measures above $\$ 50,000$ were reset to $\$ 50,000$; similarly, outpatient payment measures were top-coded at $\$ \mathbf{2 5 , 0 0 0}$. This “top-coding” affected at most eight observations for any measure.

Quality of care was measured by adherence to a) guidelineconsistent colorectal cancer screening ${ }^{23}$ and b) bone density testing for all subjects ${ }^{24}$, and c) recommended monitoring for those with cardiovascular disease (CVD) or with diabetes (DM), 
identified from ICD-9-CM diagnosis codes prior to the enrollment date.

\section{Analyses}

We used baseline interview data to compare breast cancer patients included in this study $(N=422)$ with the cases $(N=443)$ that could not be included. The groups were similar with respect to age, race/ethnicity, and a range of comorbidity, tumor and treatment characteristics.

Our key comparisons were of the 422 cases and 1,656 controls, using $t$-tests, chi-square tests, and log-rank tests (for survival data) as appropriate. We used Kaplan-Meier survival analyses, with log-rank tests for differences by group, and proportional hazards regression models to examine group differences while controlling for differences in baseline comorbidity, age, race and geography between cases and controls.

For annual utilization measures, we report $t$-test comparisons between cohorts. Analogous adjusted comparisons were tested using linear multivariate regression. Given that the cohorts were fairly well matched on important predictors, regression-based findings were very like those based on $t$ tests. Thus, we only report the latter. Quality of care measures were compared using chi-square tests.

\section{RESULTS}

Table 1 characterizes the demographics and baseline comorbidity burden of the cases and the matched controls. In both groups, over half were aged 65 to 74 ; about $4 \%$ were black; over $70 \%$ of both cohorts had less comorbidity at baseline than the Medicare average (1.0), mainly due to younger ages. While not statistically significant, more cases $(6.9 \%)$ than controls $(5.0 \%)$ were in the highest comorbidity burden category (DCG $>3.0$ ).

Most subjects ( $74 \%$ of cases and $76 \%$ of controls) were alive as of $12 / 31 / 2004$, the study end date $(p=0.16)$. Five-year survival was $80 \%$ and $82 \%$ for cases and controls, respectively ( $p=0.18$ ). Annual mortality (Fig. 1) was also similar by either log-rank test or Cox proportional hazards regression (both $p \geq 0.15$ ).

Table 1. Characteristics of the Study Population

\begin{tabular}{lcc}
\hline \multicolumn{2}{c}{ Cases $(n=422)$} & Controls $(n=1,656)$ \\
\hline Age at study inception $^{\mathrm{a}}(\%)$ & \\
$65-74$ & 54.5 & 54.3 \\
$75-84$ & 37.2 & 37.4 \\
$85+$ & 8.3 & 8.3 \\
\% Black & 4.3 & 4.4 \\
Baseline comorbidity burden score ${ }^{\mathrm{b}}$ (DCG score) \% & \\
$<1.0$ & 73.7 & 75.7 \\
1.0 & 19.4 & 19.3 \\
$>3.0$ & 6.9 & 5.0 \\
Year of study inception & & \\
1997 & 19.7 & 20.0 \\
1998 & 54.5 & 54.2 \\
1999 & 25.8 & 25.8 \\
\hline
\end{tabular}

Similarity between the two cohorts was not rejected for any of the characteristics (at 5\% significance level)

${ }^{a}$ Based on date of diagnosis for cases; for controls, the date of diagnosis for the matched case was used

${ }^{b}$ Based on the 1-year prior to study inception

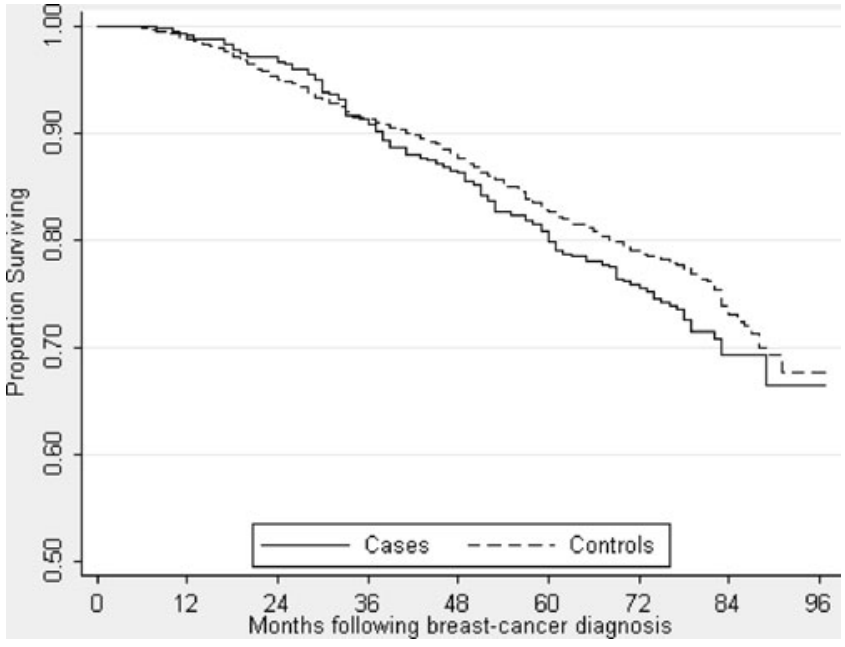

Figure 1. Survivorship of breast cancer cases and controls.

Table 2 compares healthcare utilization and comorbidity burden annually for five years following enrollment. During the first year following breast cancer diagnosis, Medicare payments for cases $(\$ 11,193)$ were significantly higher than for controls $(\$ 3,159 ; p<0.01)$. The magnitude of this difference decreased in subsequent years, yet Medicare payments remained significantly higher for cases in three of the four years. Non-breast cancer morbidity was higher for cases than controls in all years.

Table 3 characterizes utilization differences separately for inpatient and outpatient care. In the year following breast cancer diagnosis, average Medicare payments for cases were $\$ 3,935$ for inpatient care and $\$ 7,259$ for outpatient care. Both were notably higher than for controls $(\$ 1,710$ and $\$ 1,450$, respectively, $p<0.01$ ). This difference is reflected in the quantity measures: first-year hospital days averaged 3.9 for cases and 1.7 for controls $(p<0.01)$, and average number of outpatient physician visits for cases (16.6) was twice that for controls (8.1; $p<0.01)$. However, trends differed markedly for outpatient and inpatient care. The number of outpatient physician visits remained significantly higher for cases in each of the four subsequent years, although the year-five difference was smallest (cases $=11.0$; controls $=8.8, p<0.01$ ). In all five years, there were similar visit numbers for generalists and most specialists, but higher visit numbers for cancer and surgery specialists. In terms of Medicare payments, this difference was significant (in all but the fifth year). For outpatient payments by type of carephysician visits, imaging, laboratory tests and proceduresdifferences were uniform across all types. In contrast, differences in inpatient care, measured either as Medicare payments or hospital days, were not significantly different in three of the four years.

We also examined quality of care for all patients and for those with CVD or DM at study entry, which were similarly prevalent among cases and controls (Table 4). Among those with CVD, missing at least one biennial lipid test prior to the end of 2003 (for survivors) or the date of death (for nonsurvivors), was more likely than not for both cases and controls. Diabetes was also similarly prevalent (19\% vs. $17 \%$, for cases and controls, respectively), as were lipid and AIC testing, and eye examinations. Among all cases and controls, 
Table 2. Total Medicare Payments and Comorbidity Burden Following Breast Cancer Diagnosis

\begin{tabular}{|c|c|c|c|c|c|c|}
\hline \multirow[t]{3}{*}{ Period after diagnosis } & \multicolumn{3}{|c|}{ Total Medicare payments ('000 \$) } & \multicolumn{3}{|c|}{ Comorbidity burden (DCG) score } \\
\hline & \multicolumn{2}{|c|}{ Mean (Std Dev) } & \multirow[t]{2}{*}{$P$-value } & \multicolumn{2}{|c|}{ Mean (Std Dev) } & \multirow[t]{2}{*}{$P$-value } \\
\hline & Cases & Controls & & Cases & Controls & \\
\hline 1st Year & $11.2(8.3)$ & $3.2(7.2)$ & $<0.01$ & $0.90(0.84)$ & $0.80(0.68)$ & 0.01 \\
\hline 2nd Year & $3.9(7.0)$ & $3.3(7.1)$ & 0.10 & $1.04(1.01)$ & $0.96(0.87)$ & 0.12 \\
\hline 3rd Year & $5.1(8.2)$ & $3.5(7.0)$ & $<0.01$ & $1.11(1.00)$ & $0.98(0.87)$ & 0.01 \\
\hline 4th Year & $5.4(9.1)$ & $4.0(7.7)$ & $<0.01$ & 1.15 (1.03) & $1.06(1.01)$ & 0.12 \\
\hline 5th Year & $3.6(5.6)$ & 3.6 (7.9) & 0.96 & $0.95(0.76)$ & $0.95(0.82)$ & 0.90 \\
\hline
\end{tabular}

a Comorbidity burden, excluding breast cancer, is measured by diagnosis-based DCG score. Score=1 denotes mean Medicare enrollee burden $b P$-value is from a t-test for equal means in same-year measure between cases and controls

bone density testing was also equally prevalent; however, nonadherence to colorectal cancer screening among cases (33\%) was lower than that for controls $(47 \%$; $p$-value $<0.001)$.

\section{DISCUSSION}

This prospective study contrasted health outcomes and healthcare utilization for a cohort of breast cancer patients over five years following their cancer diagnosis with those of controls matched by age, geographic location and total comorbidity burden. Non-breast cancer comorbidity at baseline and five-year survival were similar in the two cohorts at baseline. Although similar in all other years, non-breast cancer comorbidity was significantly higher for cases in the first year following breast cancer diagnosis. Among survivors in each cohort, hospital utilization exhibited the same pattern, with more days of hospitalization and Medicare payments in the first follow-up year, but similar amounts subsequently. In contrast, both numbers of physician visits and total Medicare payments for outpatient services (as measured by Medicare payments) were notably higher for cases-by over 25\%-during most of the five follow-up years. Quality of care, as measured by guidelineadherent monitoring for those with baseline CVD and DM, and bone density testing for all patients, was also similar in the groups. However, colorectal cancer screening guideline adherence was better for cases.
Our estimates of utilization following breast cancer diagnosis are like those of Yabroff et al. in which Medicare payments in the first year following breast cancer diagnosis were $\$ 11,728$ (1999-2003), and higher than for a cohort matched by age and geographic location, but not comorbidity. ${ }^{25}$ While the corresponding estimate from our study was $\$ 8,034$, these figures are not directly comparable due to differences in control selection, study time-period, and geography. However, we estimated inpatient costs for our cases to be $27 \%$ of total Medicare payments for the cases, which is similar to the Yabroff et al. estimate of $25 \%$. Note that Medicare payments serve as a good proxy for inpatient and outpatient health care services as Medicare is the primary payer, accounting for over $86 \%$ of such expenditures for the elderly. ${ }^{26}$

Some studies have found greater comorbidity among cancer patients than among age-similar non-cancer patients. ${ }^{5-7}$ Therefore, when breast cancer patients are compared with non-cancer patients matched only on age and location, ${ }^{25,27,28}$ the cancer patients could have higher healthcare utilization for this reason alone. In this study we matched by baseline comorbidity in addition to age, race and geography; this enabled us to ask if the breast cancer diagnosis and treatment itself have longer term effects on healthcare utilization? While inpatient care utilization was similar between the two cohorts, numbers of physician visits and Medicare payments for outpatient care were consistently greater among breast cancer survivors-by over $100 \%$ in the first year and at least $25 \%$ in all four subsequent years. Breast cancer survivors visited

Table 3. Inpatient and Outpatient Healthcare Use Following Breast Cancer Diagnosis

\begin{tabular}{|c|c|c|c|c|c|c|}
\hline \multirow[t]{2}{*}{ Period after diagnosis } & \multicolumn{2}{|c|}{ Mean (Std Dev) } & \multirow[t]{2}{*}{$P$-value } & \multicolumn{2}{|c|}{ Mean (Std Dev) } & \multirow[t]{2}{*}{$P$-value } \\
\hline & Cases & Controls & & Cases & Controls & \\
\hline \multicolumn{7}{|l|}{ Inpatient care } \\
\hline & \multicolumn{3}{|c|}{ Inpatient Medicare payments ('000 \$) } & \multicolumn{2}{|c|}{ \# Hospitalization days } & \\
\hline 1st Year & $3.9(6.2)$ & $1.7(5.5)$ & $<0.01$ & $3.9(6.8)$ & $1.7(5.2)$ & $<0.01$ \\
\hline 2nd Year & $1.7(5.2)$ & $1.7(5.5)$ & 0.99 & $1.8(5.4)$ & $1.6(5.1)$ & 0.60 \\
\hline 3rd Year & $2.5(6.4)$ & $1.7(5.2)$ & 0.01 & $2.2(6.1)$ & $1.7(5.3)$ & 0.09 \\
\hline 4th Year & $2.4(6.6)$ & $1.9(5.7)$ & 0.17 & $2.2(6.4)$ & $1.8(5.7)$ & 0.26 \\
\hline 5th Year & $1.4(4.1)$ & $1.6(4.9)$ & 0.65 & $1.2(3.7)$ & $1.4(4.4)$ & 0.59 \\
\hline \multicolumn{7}{|l|}{ Outpatient care } \\
\hline & \multicolumn{3}{|c|}{ All outpatient Medicare payments ('000 \$) } & \multicolumn{2}{|c|}{ \# Physician Visits } & \\
\hline 1st Year & $7.3(4.8)$ & $1.5(2.2)$ & $<0.01$ & $16.6(11.1)$ & $8.1(8.5)$ & $<0.01$ \\
\hline 2nd Year & $2.2(2.7)$ & $1.5(2.3)$ & $<0.01$ & $12.5(10.1)$ & $8.6(9.1)$ & $<0.01$ \\
\hline 3rd Year & $2.6(3.1)$ & $1.8(2.6)$ & $<0.01$ & $12.3(9.9)$ & $8.9(9.7)$ & $<0.01$ \\
\hline 4th Year & $3.0(4.0)$ & $2.1(3.0)$ & $<0.01$ & $13.0(10.5)$ & $9.6(9.6)$ & $<0.01$ \\
\hline 5th Year & $2.2(2.7)$ & $2.1(1.8)$ & 0.57 & $11.0(9.5)$ & $8.8(9.2)$ & $<0.01$ \\
\hline
\end{tabular}

a P-value is from a t-test of equal mean in same-year measures between cases and controls 
Table 4. Comparison of Indicators of Inadequate Quality of Chronic Disease Care

\begin{tabular}{|c|c|c|c|}
\hline & Survivors & Controls & $P$-value \\
\hline \multicolumn{4}{|l|}{ Cardiovascular disease (CVD) } \\
\hline Baseline prevalence, \% & 49 & 49 & 0.97 \\
\hline $\begin{array}{l}\text { CVD patients who missed } \\
\text { biennial lipid test, \% }\end{array}$ & 49 & 48 & 0.87 \\
\hline \multicolumn{4}{|l|}{ Diabetes (DM) } \\
\hline Baseline prevalence, $\%$ & 19 & 17 & 0.36 \\
\hline $\begin{array}{l}\text { DM patients who missed } \\
\text { biennial lipid test, } \%\end{array}$ & 39 & 39 & 0.98 \\
\hline $\begin{array}{l}\text { DM patients who missed } \\
\text { annual AlC test, } \%\end{array}$ & 66 & 64 & 0.74 \\
\hline $\begin{array}{l}\text { DM patients who missed biennial } \\
\text { eye exam, \% }\end{array}$ & 42 & 43 & 0.80 \\
\hline \multicolumn{4}{|l|}{ (1) } \\
\hline $\begin{array}{l}\% \text { missed guideline colorectal } \\
\text { cancer screening during } \\
\text { follow-up }\end{array}$ & 21 & 27 & 0.03 \\
\hline $\begin{array}{l}\% \text { with no bone density } \\
\text { testing during follow-up }\end{array}$ & 57 & 59 & 0.47 \\
\hline
\end{tabular}

Data on care received following breast cancer diagnosis till Dec 31, 2003 a The follow-up period extends from the day after enrollment to 12/31/ 2003 or the date of death if the latter is earlier. Using the U.S. Preventive Services Taskforce guideline for colorectal cancer screening, we defined compliance for this study cohort as one of the following during the followup period (for subjects in 65-75 year age group): i) annual screening with high-sensitivity fecal occult blood testing, ii) one sigmoidoscopy examination and one fecal occult blood test, and iii) one colonoscopy examination $^{23,39}$

cancer specialists more often, but numbers of visits to mental health specialists and generalists were similar. Some of this difference may be due to a "volunteer effect", in which those who agree to enroll in a study are more likely to engage in systematic care-seeking than the controls who were selected by retrospective matching; the control group contains both women who would have and those who would not have accepted study enrollment had it been offered. ${ }^{29,30}$

Greater outpatient care among breast cancer survivors could also reflect long-term and late complications of cancer treatment, ${ }^{13,31}$ since comorbid conditions and their treatment may interact with cancer and its treatment, leading to worse physical health and higher mortality risk. ${ }^{6,32,33}$ However, survival for at least five years following diagnosis was quite similar for cases and controls, as was measured comorbidity levels in most years. Hence, more physician visits and outpatient care among breast cancer survivors does not appear to be due to their being sicker. More likely is that increased outpatient care is associated with breast cancer follow-up care. Indeed, the Institute of Medicine report From Cancer Patient to Cancer Survivor: Lost in Transition focuses on the complex follow-up needs of cancer survivors, including preventive care, monitoring for treatment side effects (e.g., adjuvant hormonal therapy) and recurrence surveillance. ${ }^{9}$

That is, breast cancer patients, after being drawn into the caregiving network, are likely to remain engaged. Several studies have examined whether breast cancer modifies careseeking for comorbid conditions among older adults. ${ }^{11,16,17,34-36}$ We examined colorectal cancer screening and bone density testing for all patients, and monitoring for two important chronic condition subgroups-those with CVD and DM. We found no differences between cases and controls, except in the case of colorectal cancer screening. Similar or better quality of care among breast cancer survivors is consistent with our finding that they had more physician visits compared to the controls. Differing study design makes comparisons with previous reports difficult. Snyder et al. found that in each of the five years of followup, breast cancer survivors had less colorectal cancer screening, bone density and lipid testing than matched controls. ${ }^{36}$ However, their controls were chosen from women who had had a mammogram during the baseline year, making it likely that their overall quality of care was also above average. In contrast, Earle et al. found that breast cancer survivors with diabetes had higher rates of lipid testing than matched controls. ${ }^{17}$ Since comorbidity was not a matching criterion, this could have been due to differences in comorbid disease burden. Ultimately it has not been clear whether breast cancer survivors receive either more or less chronic disease care than similarly-ill women with the same morbidity burden. Keating et al., which also matched controls by comorbidity, found any-cancer survivors with diabetes receiving diabetes screening "of generally similar quality" as non-cancer diabetics. ${ }^{16}$ This is consistent with our findings.

Previous studies have examined breast cancer survivors retrospectively; ${ }^{17,37}$ in contrast, we have been able to examine survival prospectively. The estimated five-year survival for this breast cancer cohort $(79.6 \%)$, is lower than that for National Cancer Institute's Surveillance Epidemiology and End Results (SEER) regions $\left(89.6 \%\right.$; 1996-2003). ${ }^{38}$ This could partly be due to the difference in populations represented-our subjects were from four selected geographic areas, while SEER data is obtained from areas containing over $26 \%$ of the national population. Further, because we were also interested in studying utilization, we examined only fee-for-service Medicare beneficiaries.

This study has several limitations. The study population was clustered in four geographic areas and may not generalize nationally. The breast cancer cohort had volunteered for a study; they may be healthier, or more prone to positive health behaviors, than other survivors, or a matched cohort selected from the general population. ${ }^{29,30}$ Also, we used only Medicare administrative data, with limited clinical detail on comorbidity severity and no pharmacy data; since patient comorbidity was identified from diagnoses coded on claim forms, breast cancer survivors' greater interaction with health care providers may partly explain their higher measured comorbidity. Both cases and controls participated in Medicare FFS; in this sense they were similar, but not fully representative of Medicare enrollees, upwards of $20 \%$ of whom are enrolled in managed care plans. Also, since studying survivorship requires waiting for the data to mature, our subjects' care does not reflect recent advances in breast cancer management, such as sentinel lymph node biopsy and the use of aromatase inhibitors. We also recognize that the sub-cohorts examined for quality of care, involving those with baseline CVD and diabetes, were small. The study cohort has few minorities ( $4.3 \%$ black), a result of a) the population distribution of the study regions and b) older black women being at lower risk of developing breast cancer, but at higher risk of presenting with late stage disease (and therefore excluded from our study). Finally, due to lack of completeness of provider specialty field, the provider type of a sizable proportion of physician visits (about 25\%) could not be determined.

In summary, this study clarifies the mixed picture related to longitudinal health care for older breast cancer survivors. We found that, beyond the first year after breast cancer diagnosis, older survivors have patterns of disease burden, inpatient care and quality of care for other health problems quite similar to 
those of women without breast cancer. There was one exception-they had notably more physician visits, especially for cancer and surgical specialists, than non-breast-cancer patients in each of five years of follow-up. Reasons for this should be pursued using more detailed clinical data. For example, were the excess visits for cancer surveillance, and, if so, did they follow accepted guidelines?

Acknowledgements: This work was supported by grants CA106979, CA/AG 70818, CA84506, and CA92395 from the National Cancer Institute.

Conflict of Interest: None disclosed.

Corresponding Author: Amresh D. Hanchate, $\mathrm{PhD}$; Health Care Research Unit, Section of General Internal Medicine, Boston University School of Medicine, 801, Massachusetts Ave \#2077. Boston, MA 01532, USA (e-mail: hanchate@bu.edu).

\section{REFERENCES}

1. American Cancer Society. Breast Cancer: Facts \& Figures. 2007.

2. Berry DA, Cronin KA, Plevritis SK, et al. Effect of screening and adjuvant therapy on mortality from breast cancer. $\mathrm{N}$ Engl $\mathrm{J}$ Med. 2005;353(17): 1784-92

3. Natinal Cancer Institute. Probability of Breast Cancer in American Women. 2009. http://www.cancer.gov/cancertopics/factsheet/Detec tion/probability-breast-cancer. Published Last Modified Date|. Accessed May 2010|.

4. Guralnik JM. Assessing the impact of comorbidity in the older population. Ann Epidemiol. 1996;6(5):376-80.

5. Ogle KS, Swanson GM, Woods N, Azzouz F. Cancer and comorbidity: redefining chronic diseases. Cancer. 2000;88(3):653-63.

6. Smith AW, Reeve BB, Bellizzi KM, et al. Cancer, comorbidities, and health-related quality of life of older adults. Health Care Financ Rev. 2008;29(4):41-56.

7. Yancik R, Havlik RJ, Wesley MN, et al. Cancer and comorbidity in older patients: a descriptive profile. Ann Epidemiol. 1996;6(5):399-412.

8. Keating NL, Norredam M, Landrum MB, Huskamp HA, Meara E. Physical and mental health status of older long-term cancer survivors. J Am Geriatr Soc. 2005;53(12):2145-52.

9. Hewitt ME, Greenfield S, Stovall E, National Cancer Policy Board (U.S.). Committee on Cancer Survivorship: Improving Care and Quality of Life. From Cancer Patient to Cancer Survivor: Lost in Transition. Washington: National Academies Press; 2006.

10. Snyder CF, Earle CC, Herbert RJ, Neville BA, Blackford AL, Frick KD. Preventive care for colorectal cancer survivors: a 5-year longitudinal study. J Clin Oncol. 2008;26(7):1073-9.

11. Burstein HJ, Winer EP. Primary care for survivors of breast cancer. N Engl J Med. 2000;343(15):1086-94.

12. Hurria A, Hudis C. Follow-up care of breast cancer survivors. Crit Rev Oncol Hematol. 2003;48(1):89-99.

13. Partridge AH, Winer EP, Burstein HJ. Follow-up care of breast cancer survivors. Semin Oncol. 2003;30(6):817-25.

14. Earle CC. Failing to plan is planning to fail: improving the quality of care with survivorship care plans. J Clin Oncol. 2006;24(32):5112-6.

15. Institute of Medicine. From cancer patient to cancer survivor: Lost in transition. Washington: National Academy Press: 2005.
16. Keating NL, Zaslavsky AM, Herrinton LJ, Selby JV, Wolf RE, Ayanian JZ. Quality of diabetes care among cancer survivors with diabetes. Med Care. 2007;45(9):869-75.

17. Earle CC, Burstein HJ, Winer EP, Weeks JC. Quality of non-breast cancer health maintenance among elderly breast cancer survivors. J Clin Oncol. 2003;21(8):1447-51.

18. Silliman RA, Guadagnoli E, Rakowski W, et al. Adjuvant tamoxifen prescription in women 65 years and older with primary breast cancer. J Clin Oncol. 2002;20(11):2680-8.

19. DxCG Inc. DxCG RiskSmart: Clinical Classifications Guide. Boston: DxCG; 2007.

20. Ash AS, Ellis RP, Pope GC, et al. Using diagnoses to describe populations and predict costs. Health Care Financ Rev. 2000;21(3):7-28.

21. Ash AS, Posner MA, Speckman J, Franco S, Yacht AC, Bramwell L. Using claims data to examine mortality trends following hospitalization for heart attack in Medicare. Health Serv Res. 2003;38(5): 1253-62.

22. Brown ML, Riley GF, Schussler N, Etzioni R. Estimating health care costs related to cancer treatment from SEER-Medicare data. Med Care. 2002;40(8 Suppl):IV-104-17.

23. Whitlock EP, Lin JS, Liles E, Beil TL, Fu R. Screening for colorectal cancer: a targeted, updated systematic review for the U.S. Preventive Services Task Force. Ann Intern Med. 2008;149(9):638-58.

24. U.S. Preventive Services Task Force. Screening for Osteoporosis in Postmenopausal Women: Recommendations and Rationale. Rockville: Agency for Healthcare Research and Quality; 2002.

25. Yabroff KR, Lamont EB, Mariotto A, et al. Cost of care for elderly cancer patients in the United States. J Natl Cancer Inst. 2008;100 (9):630-41.

26. Centers for Medicare \& Medicaid Services. National Health Expenditures Fact Sheet: U.S. Department of Health \& Human Services; 2009.

27. Warren JL, Brown ML, Fay MP, Schussler N, Potosky AL, Riley GF. Costs of treatment for elderly women with early-stage breast cancer in fee-for-service settings. J Clin Oncol. 2002;20(1):307-16.

28. Warren JL, Yabroff KR, Meekins A, Topor M, Lamont EB, Brown ML. Evaluation of trends in the cost of initial cancer treatment. J Natl Cancer Inst. 2008;100(12):888-97.

29. Froom P, Melamed S, Kristal-Boneh E, Benbassat J, Ribak J. Healthy volunteer effect in industrial workers. J Clin Epidemiol. 1999;52(8):731-5.

30. Heilbrun LK, Nomura A, Stemmermann GN. The effects of nonresponse in a prospective study of cancer: 15-year follow-up. Int $\mathrm{J}$ Epidemiol. 1991;20(2):328-38.

31. Peppercorn J, Partridge A, Burstein HJ, Winer EP. Standards for follow-up care of patents with breast cancer. Breast. 2005;14(6):500-8.

32. Ahern TP, Lash TL, Thwin SS, Silliman RA. Impact of acquired comorbidities on all-cause mortality rates among older breast cancer survivors. Med Care. 2009;47(1):73-9.

33. Nagel G, Wedding U, Rohrig B, Katenkamp D. The impact of comorbidity on the survival of postmenopausal women with breast cancer. J Cancer Res Clin Oncol. 2004;130(11):664-70.

34. Duffy CM, Clark MA, Allsworth JE. Health maintenance and screening in breast cancer survivors in the United States. Cancer Detect Prev. 2006;30(1):52-7.

35. Snyder CF, Frick KD, Kantsiper ME, et al. Prevention, screening, and surveillance care for breast cancer survivors compared with controls: changes from 1998 to 2002. J Clin Oncol. Jan 212009.

36. Snyder CF, Frick KD, Peairs KS, et al. Comparing care for breast cancer survivors to non-cancer controls: a five-year longitudinal study. J Gen Intern Med. Jan 212009.

37. Earle CC, Neville BA. Under use of necessary care among cancer survivors. Cancer. 2004;101(8):1712-9.

38. Ries L, Melbert D, Krapcho M, et al. SEER Cancer Statistics Review, 1975-2004. Bethesda, MD; 2007.

39. Ananthakrishnan AN, Schellhase KG, Sparapani RA, Laud PW, Neuner JM. Disparities in colon cancer screening in the Medicare population. Arch Intern Med. 2007;167(3):258-64. 